\title{
EP-118
}

\section{Anticipatory extended cholecystectomy}

\author{
Mukteshwar DASARI, Vinay Kumar KAPOOR*
}

Department of Surgical Gastroenterology, SGPGIMS, Lucknow, India

Introduction: Gall stones (GS) cause chronic cholecystitis (CC) and xantho-granulomatous cholecystitis (XGC) resulting in thickwalled GB (TWGB). Gall bladder cancer (GBC) also presents as TWGB. CC and XGC are treated with simple cholecystectomy, GBC merits extended cholecystectomy (EC). We propose a surgical approach, anticipatory extended cholecystectomy (AEC), for TWGB. AEC does not violate cholecysto-hepatic plane and does not ruin chances of cure in GBC.

Methods: AEC involves cholecystectomy with 2-cm wedge of liver, followed by frozen section histological examination. Lymphadenectomy is added if GBC is confirmed. AEC was performed in 49 patients with TWGB between 2011 and 2020.

Results: GBC was suspected on US in 44 and on computed tomography (CT) in 37 patients. FNAC in 9 patients ( 3 by us and 6 elsewhere) showed neoplastic cells 1, suspicious for malignancy 1, atypical cells 2, XGC 3 and negative for neoplastic cells 2. Preoperative suspicion of GBC was in 13 cases. Frozen section was done in 42 cases (inconclusive in 1), not done in 5 and not available in 2 cases. Lymphadenectomy was done in 5 cases (FNAC showing neoplastic cells 1, frozen GBC 2, suspicious 1, and inconclusive 1). Final histopathology revealed adenocarcinoma in 3, adenocarcinoma with XGC in 1, XGC with adenocarcinoma in 1, CC in 9, benign GB polyps in 2 and XGC in 33.

Conclusions: In TWGB on US/CT with low suspicion of cancer, AEC serves as triage-if frozen section shows GBC, AEC can be completed to EC by adding lymphadenectomy. We have named AEC as 'Lucknow' approach for TWGB. 